\title{
Remoção cirúrgica de sialolito em glândula submandibular: relato de caso
}

Surgical removal of sialolite in submandibular gland: case report

Remoción quirúrgica de sialolito en glándula submandibular: relato de caso

Luiz Henrique Soares TORRES
Marcelo Soares dos SANTOS

Jiordanne Araújo DINIZ ${ }^{2}$

Caio Pimenteira UCHÔA ${ }^{3}$

Joana de Ângelis Alves SILVA ${ }^{4}$

Valfrido Antônio PEREIRA FILHO ${ }^{1}$

Emanuel Dias de OLIVEIRA e SILVA ${ }^{3}$

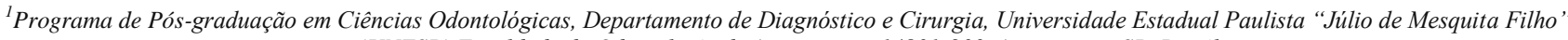
(UNESP) Faculdade de Odontologia de Araraquara, 14801-903, Araraquara-SP, Brasil

${ }^{2}$ Programa de Pós-graduação em Cirurgia e Traumatologia Bucomaxilofacial, Hospital Universitário Oswaldo Cruz, UPE Universidade de Pernambuco, 50100-130 Recife-PE, Brasil

${ }^{3}$ Departamento de Cirurgia e Traumatologia Bucomaxilofacial, Hospital Universitário Oswaldo Cruz, UPE Universidade de Pernambuco, 50100-130 Recife-PE, Brasil. ${ }^{4}$ Cirurgiã-Dentista pela Faculdade de Odontologia de Pernambuco, UPE Universidade de Pernambuco 50100-130 Recife, Brasil

\section{Resumo}

Introdução: A sialolitíase é a desordem mais comum das glândulas salivares acometendo principalmente a glândula submandibular e o seu ducto. Caracteriza-se pela formação de cálculos ou sialólitos no interior do ducto ou do próprio parênquima glandular. A etiologia pode estar associada ao $\mathrm{pH}$ alcalino e aumento da concentração de cálcio na saliva, além de fatores anatómicos como estenose ou diverticulação do ducto salivar. Objetivo: relatar um caso clínico de sialólito localizado no ducto da glândula submandibular, bem como discutir a literatura pertinente sobre o tema. Métodos: Paciente do gênero masculino, compareceu ao Hospital Geral de Areias com queixa de aumento em região de soalho bucal direito. Foram solicitadas radiografias panorâmica e oclusal e observou-se massa radiopaca de formato cilíndrico e alongado na região submandibular. A excisão cirúrgica foi realizada sob anestesia local. Paciente não apresentou complicações e retomou-se o reestabelecimento da patência do ducto e normalidade do fluxo salivar. Conclusões: A conduta mostrou-se eficaz no manejo da lesão através de remoção cirúrgica por acesso intrabucal. Estas desordens são facilmente diagnosticadas através do exame clínico e de imagem.

Descritores: Glândula Submandibular; Soalho Bucal; Cálculos das Glândulas Salivares.

\section{Abstract}

Introduction: Sialolithiasis is the most common disorder of the salivary glands affecting mainly the submandibular gland and its duct. Characterized by the formation of calculi or sialolites inside the duct or the glandular parenchyma itself. The etiology may be associated with alkaline $\mathrm{pH}$ and increased calcium concentration in saliva, in addition to anatomical factors such as stenosis or diverticulation of the salivary duct. Objective: to report a clinical case of sialolith located in the duct of the submandibular gland, as well as to discuss pertinent literature on the subject. Methods: Patients of the male gender, attended the General Hospital of Areias complaining of an increase in the region of the right buccal floor. Panoramic and occlusal radiographs were requested and a radiopaque mass of cylindrical and elongated shape was observed in the submandibular region. Surgical excision was performed under local anesthesia. Patient did not present complications and the reestablishment of duct patency and normal salivary flow was resumed. Conclusions: The conduct was effective in the management of the lesion through surgical removal by intraoral access. These disorders are easily diagnosed through clinical and imaging examination.

Descriptors: Submandibular Gland; Mouth Floor; Salivary Gland Calculi.

\section{Resumen}

Introducción: La sialolitiasis es el desorden más común de las glándulas salivares que afectan principalmente a la glándula submandibular y su conducto. Se caracteriza por la formación de cálculos o sialolitos en el interior del conducto o del propio parénquima glandular. La etiología puede estar asociada al pH alcalino y aumento de la concentración de calcio en la saliva, además de factores anatómicos como estenosis o diverticulación del conducto salivar. Objetivo: relatar un caso clínico de sialolito localizado en el conducto de la glándula submandibular, así como discutir la literatura pertinente sobre el tema. Métodos: Paciente del género masculino, acudió al Hospital General de Areias con queja de aumento en región de suelo bucal derecho. Se solicitaron radiografías panorámicas y oclusales y se observó masa radiopaca de formato cilíndrico y alargado en la región submandibular. La excisión quirúrgica se realizó bajo anestesia local. El paciente no presentó complicaciones y se reanudó el restablecimiento de la patencia del conducto y la normalidad del flujo salivar. Conclusiones: La conducta se mostró eficaz en el manejo de la lesión mediante la remoción quirúrgica por acceso intraoral. Estos desórdenes son fácilmente diagnosticados a través del examen clínico y de imagen.

Descriptores: Glándula Submandibular, Suelo de la Boca, Cálculos de las Glándulas Salivales.

\section{INTRODUÇÃO}

A sialolitíase é a patologia mais comum encontrada nas glândulas salivares e é um dos maiores distúrbios obstrutivos das glândulas salivares maiores, sendo causada por cálculos na própria glândula e no seu ducto ${ }^{1,2}$. A glândula submandibular é acometida em $83 \%$ dos casos, seguida pela glândula parótida com $10 \%$, sublingual e salivar menor em $7 \% 3$. O tamanho do cálculo pode variar de menos de $1 \mathrm{~mm}$ a alguns centímetros ${ }^{1,4}$, embora a maioria se apresente menor que $10 \mathrm{~mm}$. Existem alguns relatos de cálculos maiores que $15 \mathrm{~mm}$, entretanto são de ocorrência rara ${ }^{1}$.

O diagnóstico desta condição na glândula submandibular é feito por meio da associação da inspeção e palpação do soalho bucal e da região submandibular. A confirmação da presença do sialólito é realizada por exames de imagem como radiografias panorâmicas e oclusais, ultrassonografia, sialografias, tomografia computadorizada e ressonância nuclear magnética ${ }^{5,6}$. O paciente que apresenta esta condição pode referir dores e edema no trajeto do ducto ou na glândula envolvida dependendo do local da obstrução, associados à alimentação e subsequente sialoadenite ${ }^{2,5,7}$.

O tratamento pode variar de acordo com a localização e o tamanho do cálculo. Para alívio dos sintomas associados, são instituídas terapias com antibióticos, analgésicos ou antipiréticos. Quando o cálculo se encontra no interior do ducto glandular, o tratamento mais comumente escolhido é a remoção 
do cálculo através do acesso intrabucal. Quando o cálculo estiver localizado no interior da glândula ou porção proximal do ducto, faz-se necessária a sialodenectomia. Esta consiste na remoção completa da glândula submandibular ${ }^{5,8}$. Porém outras terapias como o uso de sialogogos com massagem glandular para promover a salivação e irrigar os pequenos sialólitos para o orifício do ducto, a litotripsia que fragmenta o cálculo por meio de ondas de choque e o uso de laser de dióxido de carbono (CO2) podem ser empregadas ${ }^{2,5}$.

O objetivo deste artigo é relatar a remoção cirúrgica de um sialólito no interior do ducto de Wharton, com o diagnóstico feito através de exame clínico e radiografias panorâmica e oclusal, bem como discutir a literatura pertinente sobre o tema.

\section{CASO CLÍNICO}

Paciente do gênero masculino, compareceu ao Hospital Geral de Areias (Recife/PE) com queixa de "aumento em região de fundo da boca" com evolução de seis meses. Ao exame físico intrabucal, notou-se aumento de volume em região de assoalho bucal direito, endurecido à palpação, de coloração amarelada e drenagem purulenta ativa (Figura 1A). Solicitou-se exames complementares de imagem (radiografia panorâmica e oclusal), onde pode-se observar massa radiopaca de formato cilíndrico e alongado na região submandibular direita (Figura 2A e B). Sob anestesia local, foi realizada a excisão cirúrgica do sialólito (Figura 1B e C).

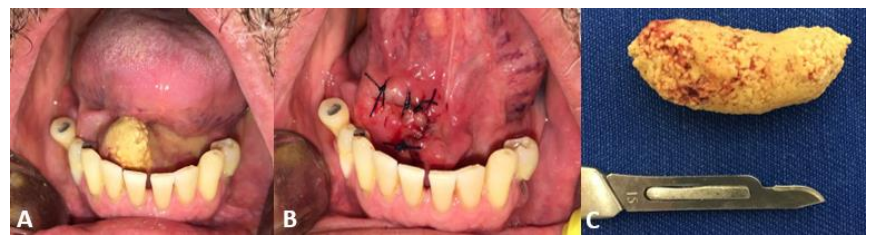

Figura 1: A: Aspecto clínico inicial e presença de secreção purulenta; B: pós-cirúrgico imediato. C: peça clínica.

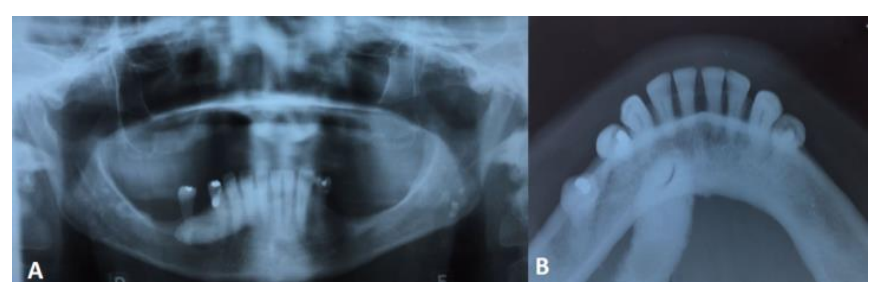

Figura 2: Aspecto radiográfico. A: Radiografia panorâmica; B: Radiografia oclusal (notar massa nodular radiopaca à direita).

Paciente recebeu alta no mesmo dia, com prescrição de antibióticos e analgésicos (amoxicilina 500mg, 01 cápsula de 8/8 horas, por 07 dias e dipirona 500mg, 01 comprimido de $6 / 6 \mathrm{~h}$, por 02 dias), além de orientação das condutas pósoperatórias como dieta e higiene local. Após sete dias, paciente retornou ao Hospital sem queixas ou complicações. As suturas foram removidas. Ao exame físico intrabucal, notou-se o restabelecimento da patência do ducto e normalidade do fluxo salivar (Figura 3).

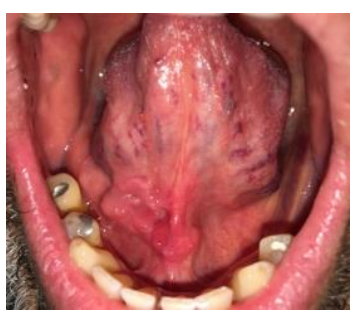

Figura 3: Aspecto clínico pós-cirúrgico após 21 dias. Patência do ducto reestabelecida.

Os sialólitos são formados em duas fases, a primeira pela precipitação de cristais depositados sob uma substância orgânica e a segunda, pela deposição orgânica e material não orgânico. Por seguinte, a mineralização é conduzida pelo acúmulo de cálcio e alteração do $\mathrm{pH}$, que, então, reduz a solubilidade do fosfato de cálcio na saliva. O cálculo consiste em uma mistura heterogênea de diferentes fosfatos de cálcio, sendo o seu componente mais comum a apatita .

O aumento da alcalinidade da saliva, infecção ou inflamação ou até mesmo um trauma físico no ducto ou glândula são fatores predisponentes à formação do cálculo. A alta incidência da sialolitíase na glândula submandibular pode ser explicada pelo pH alcalino, secreção mucosa e alta concentração de cálcio desta glândula. Além disso, outro fator contribuinte é ducto longo, que circunda o músculo milohioídeo e a secreção no sentido antigravitacional $^{9}$. Assim, a obstrução das glândulas salivares pela presença de um sialólito representa uma entidade patológica multifatorial, por conta da viscosidade da saliva, do tamanho e anatomia do conduto que possuem uma grande importância na predisposição desta alteração ${ }^{10}$. A alta prevalência associada à glândula submandibular deve-se à anatomia tortuosa e longa do ducto Wharton que possui uma direção anterior e superior para o soalho bucal além de ter o calibre menor próximo a carúncula ${ }^{4}$. Diferentemente, o ducto de Stensen possui um trajeto retilíneo, uniforme e a ajuda dos músculos da mastigação, que o sobrepõe, no fluxo salivar ${ }^{4}$.

O aumento de volume e dor da glândula afetada, principalmente nos horários de refeições é um sinal clínico muito comum e a sua intensidade depende do grau de obstrução e a quantidade de pressão produzida no interior da glândula. Pequenos sialólitos que permitem a passagem da saliva através do ducto podem ser assintomáticos, enquanto que os de tamanhos maiores podem obstruir a passagem da saliva causando dor, inchaço e infecção da glândula envolvida ${ }^{11}$. Neste caso o paciente relatava aumento de volume na região de assoalho bucal e sintomatologia dolorosa. A hipótese de diagnóstico do presente caso foi obtida através do exame intrabucal, da "ordenha" glandular e da radiografia panorâmica e do tipo oclusal. 
São descritos diversos métodos de diagnóstico, porém a radiografia convencional oclusal é a mais indicada para detectar sialólitos, sendo estas imprescindíveis para demonstrar os cálculos radiopacos, uma vez que, é muito incomum possuírem a combinação de massas radiopacas e radiolúcidas ${ }^{4,10}$. Já a sialografia está indicada no caso de sinais de sialoadenite, cálculos radiolúcidos ou cálculos submandibulares e parotídeos profundos, sendo contraindicados no caso de infecção ou pacientes com alergia ao meio de contraste ${ }^{12}$.

Alguns fatores influenciam o manejo dessa condição, dentre eles pode-se citar a glândula é afetada, o tamanho e a localização do cálculo, visto que o diâmetro do sialólito e a localização no parênquima são fatores que aumentam a dificuldade no tratamento da sialolitíase. É pressuposto que, sempre que possível, deve-se optar pelo método mais conservador ou o que mais se adeque à situação específica para o paciente ${ }^{9,12}$. O tratamento conservador deve ser reservado para sialólitos de pequeno diâmetro e localização superficial, utilizando massagens leves das glândulas, uso de sialogogos, calor úmido e aumento da ingestão de líquido 9. Assim sendo, a remoção cirúrgica do sialólito via acesso intrabucal é realizada principalmente quando o cálculo se encontra no terço distal da glândula, podendo ser facilmente palpado. É feito uma incisão no assoalho da boca, expõe-se e incisa o ducto afetado. Dessa forma, o cálculo é visualizado e removido. $\mathrm{O}$ ducto é então suturado à mucosa oral, deixando-o aberto para adequada drenagem. Deve-se atentar para a possibilidade de a glândula estar infectada, nestes casos, deve-se instituir antibioticoterapia coadjuvante ao tratamento $^{12}$.

O prognóstico é favorável, embora deva-se levar em consideração a localização, tamanho e quantidade de sialolitos. Após a eliminação dos cálculos, deve-se fazer a estimulação contínua com sialogogos como suco de limão, ácido ascórbico e goma de mascar. Os sialolitos podem apresentar recidiva ou, em caso de persistência da obstrução, causar lesão do componente parenquimatoso da glândula e causar sialoadenite; no entanto, na maioria dos casos, as glândulas afetadas funcionam normalmente após a remoção da lesão ${ }^{10}$.

\section{CONSIDERAÇÕES FINAIS}

O sinal clínico bastante evidente do sialólito na glândula submandibular é a tumefação e dor no assoalho bucal, sendo o diagnóstico estabelecido através da associação entre o exame clínico e radiográfico. A escolha do tratamento pelo profissional está diretamente ligada à localização do cálculo salivar e sua dimensão, onde pode-se optar por uma terapia não invasiva ou intervenção cirúrgica. No presente caso, o tratamento proposto por meio de remoção cirúrgica com acesso intraoral se mostrou eficaz e sem nenhuma complicação.

\section{REFERÊNCIAS}

1. Ribeiro J, Mourão CF, Magacho LF, Albieiri F, Vicentis G, Fernandes O, et al. Tratamento cirúrgicos dos sialolitos gigantes das glândulas submandibulares: relato de dois casos. In J Sci Dent. 2016;22(46).

2. Acevedo JAD, Trocino BS, Mercado HR. Sialolitiasis del conducto submandibular: reporte de un caso reporte de un caso. Rev Mex Cir Bucal Maxilofac. 2017;13(3):100-7.

3. França DCC, Facio JC, Maia CMP, Monteiro AD, Carvalhosa AA, Moi GP et al. Sialolito no ducto da glândula submandibular: relato de casa. Connection Line. 2012;7:78-89.

4. Landgraf H, Assis AF, Klüppel LE, Oliveira CF, Grabielli MAC. Extenso sialolito no ducto da glândula submandibular: relato de caso Rev Cir Traumatol Buco-Maxilo-Fac. 2006;6(2):29-34.

5. Kim JH, Aoki EM, Rodriguez A, Cortes ARG, Abdala-Júnior R, Asaumi J, Arita ES. Comparison of the diagnostic performance of panoramic and occlusal radiographs in detecting submandibular sialoliths. Imaging Sci Dent. 2016;46(2):87-92.

6. Tahher AA. The incidence and composition of salivary stones (sialolithiasis) in Iran: analysis of 95 cases - a short report. Singapore Dent J. 1990:14(1):33-5.

7. Cho S, Han J, Kim J, Lee S, Jo J, Kim C et al. Removal of submandibular calculi by surgical method and hydraulic power with curved needle : a case report. J Korean Assoc Oral Maxillofac Surg. 2017;43(3):182-85.

8. Pereira RS, Bonardi JP, Silva JR, Mourão CFAB, Souza FI, Pimentel RM. Remoção cirúrgica de sialolito no ducto da glândula submandibular: relato de caso. Arch Health Invest. 2017;6(4):185-88.

9. Folchini S, Stolz AB. Sialolitos na glândula submandibular: relato de caso. Odontol ClínCient. 2016;15(1):67-71.

10.Ogura I, Hayama K, Sue M, Oda T, Sasaki Y. Submandibular sialolithiasis with CT and scintigraphy: CT values and salivary gland excretion in the submandibular glands. Imaging Sci Dent. 2017;47(4):227-32.

11. Alves NS, Soares GG, Azevedo RS, Camisasca DR. Sialolito de grandes dimensões no ducto da glândula 
submandibular. Rev Assoc Paul Cir Dent. 2014;68(1):49-53.

12.Baurmash HD. Submandibular salivary stones: current management modalities. J Oral Maxillofac Surg. 2004;62(3)369-78.

\section{CONFLITO DE INTERESSES}

Os autores declaram não haver conflitos de interesse.

AUTOR PARA CORRESPONDENCIA

Luiz Henrique Soares Torres

luiz-lhst@hotmail.com

Submetido em 07/02/2019

Aceito em 24/05/2019 